\title{
Comparison of 16-channel laser photoreceivers for topographic mapping Invited paper
}

\author{
Michael A. Krainak, Guangning Yang, Xiaoli Sun, Wei Lu \\ NASA Goddard Space Flight Center \\ Xiaogang Bai, Ping Yuan, Paul McDonald, Joseph Boisvert, \\ Robyn Woo, Kam Wan, Rengarajan Sudharsanan \\ Boeing Spectrolab \\ Verle Aebi, Derek Sykora, Ken Costello \\ Intevac Inc.
}

\begin{abstract}
Topographic mapping lidar instruments must be able to detect extremely weak laser return signals from high altitudes including orbital distance. The signals have a wide dynamic range caused by the variability in atmospheric transmission and surface reflectance under a fast moving spacecraft. Ideally, lidar detectors should be able to detect laser signal return pulses at the single photon level and produce linear output for multiple photon events. Silicon avalanche photodiode (APD) detectors have been used in most space lidar receivers to date. Their sensitivity is typically hundreds of photons per pulse, and is limited by the quantum efficiency, APD gain noise, dark current, and preamplifier noise. NASA is pursuing three approaches for a 16-channel laser photoreceiver for use on the next generation direct-detection airborne and spaceborne lidars. We present our measurement results and a comparison of their performance.
\end{abstract}

\section{INTRODUCTION}

In 2007 the National Research Council (NRC), responded to requests from the National Aeronautics and Space Administration (NASA) Office of Earth Science, the National Oceanic and Atmospheric Administration (NOAA) National Environmental Satellite Data and Information Service (NESDIS), and the U.S. Geological Survey (USGS) Geography Division to conduct a decadal survey to generate consensus recommendations from the Earth and environmental science and applications communities regarding a systems approach to space-based and ancillary observations that encompasses the research programs of NASA; the related operational programs of NOAA; and associated programs such as Landsat, a joint initiative of USGS and NASA. The NRC recommended a total of fifteen missions ${ }^{1}$ with benefits range from information for short-term needs, such as weather forecasts and warnings for protection of life and property, to the longer-term scientific understanding necessary for future applications that will benefit society in ways still to be realized. Three out of the fifteen recommended missions are laser based instrument for topography mapping of the Earth's surface. These are the Ice, Cloud, and Land Elevation Satellite-II (ICESat-II), Deformation, Ecosystem Structure and Dynamics of Ice (DESDynl) tier-1 missions and Lidar Surface Topography (LIST) as a third tier mission. The DESDynI lidar was recently cancelled. However, a similar instrument is being proposed to the Earth Venture-2 (International Space Station) opportunity. These three missions could all possibly benefit from a very low-noise (near single-photon sensitive), high-bandwidth $(1 \mathrm{GHz})$, near-infrared optical receiver. Improvements in detector quantum efficiency translate directly to reduced laser energy requirements for active laser instruments. This minimizes spacecraft resource requirements (mass, power, volume) and greatly enhances laser and system reliability.

All of NASA's space-based laser altimetry missions to date have used versions of the near-infrared enhanced silicon avalanche photodiode (APD) detector manufactured by PerkinElmer Opto-Electronics Canada (a.k.a. EG\&G and formerly RCA) for detecting laser signal returns at $1064 \mathrm{~nm}$ wavelength. These missions include NEAR, 
CLEMENTINE, MOLA, ICESAT/GLAS, CALIPSO, MLA and LOLA. The spaceflight optical receivers are based on custom-built improved versions of the commercial Model C30954E APD. Its RF bandwidth of $140 \mathrm{MHz}$ is matched to the laser transmitter pulse bandwidth. The Si APDs exhibit low excess noise factor and low noise (NEP of 40 50 $\mathrm{fW} / \mathrm{rt}(\mathrm{Hz})$ over a bandwidth of $140 \mathrm{MHz}$ ). This silicon APD-based optical receiver is described in detail in recent publications [2,3]. A recent result [4] was an NEP of $5 \mathrm{fW} / \mathrm{rt}(\mathrm{Hz})$ with $200 \mathrm{MHz}$ bandwidth.

The laser altimeter system RF signal bandwidth is designed [5] for a specific spatial resolution and average surface topography roughness. For the space-based ICESat-GLAS instrument, these parameters were 70-meter diameter ground area, surface slope varying from $0-30$ degrees and $140 \mathrm{MHz}$ RF signal bandwidth (corresponding to the $(-20 \mathrm{~dB})$ bandwidth for a 6 ns laser transmitter pulse width). NASA's future laser altimeters plan to have a higher spatial resolution. A topographic mapping spatial resolution of 5 meters is planned for the LIST mission. This increases the $\mathrm{RF}(-20 \mathrm{~dB})$ signal bandwidth to greater than $1 \mathrm{GHz}$ and reduces the corresponding laser pulse width to less than $1 \mathrm{~ns}$. The $1 \mathrm{~ns}$ pulse width also provides higher height resolution and increased probability of ground location detection under trees for reasonably flat $(<3$ degree surface slope) regions. Si photoreceivers do not meet these requirements. At 1.06 $\mu \mathrm{m}$, in order to get reasonable absorption, a very thick absorber (several tens of microns) is necessary. The thick absorber limits the Si APD bandwidth to less than a few hundred MHz. On the other hand, III-V compound semiconductor based APDs, like InGaAs APDs, show high quantum efficiency in the 1 to $1.6 \mu \mathrm{m}$ wavelength region and fast response [2]; bandwidths greater than several $\mathrm{GHz}$ are commonly achieved with these APDs. However, current InGaAs APDs have NEP higher than $>500 \mathrm{fW} / \sqrt{\mathrm{Hz}}$ due to the use of high excess noise InP layer as a multiplier.

There are several reasons that make it difficult to extend the near-infrared-enhanced-silicon APD RF bandwidth to 1 $\mathrm{GHz}$. First, a minimum diameter is required to match the laser ground footprint diameter image size for a practical telescope optics f-number. This leads to a larger device capacitance that contributes to the limiting the maximum bandwidth. Second, increasing the device thickness to possibly further enhance the absorption length is not fruitful because it increases the noise current and it increases the electron drift time. Therefore a new detector is required for future NASA lidar missions that require $1 \mathrm{GHz} \mathrm{RF}$ bandwidth.

In 2009, we proposed and were awarded a NASA Earth Science Technology Organization (ESTO) Advanced Component Technology (ACT) program to pursue new detector development. Our idea is to use the impact ionization engineered $\left(I^{2} E\right)$ material system in Indium Aluminum Arsenide (InAlAs) to simultaneously achieve high near-infrared quantum efficiency $(75 \%)$, large diameter $(200 \mathrm{~mm}),>1 \mathrm{GHz}$ bandwidth and high sensitivity (low-noise -300 $\mathrm{fW} / \sqrt{\mathrm{Hz}}$ ). In 2007, we began work on an Instrument Incubator Program (IIP) to advance and demonstrate needed technologies for the LIST mission with the Airborne LIST Simulator (A-LISTS) pathfinder instrument [6]. A-LISTS is a micropulse, waveform recording system that is based on a new, and highly efficient laser measurement approach utilizing emerging laser transmitter and the $I^{2} E$ APD and intensified photodiode (IPD) detector technologies described here.

\section{LOW EXCESS NOISE APDS}

APDs convert light signals into electrical signals with a gain from an avalanche process. Both photo generated holes and electrons could initiate an avalanche process. The excess noise could be characterized by $k=\alpha / \beta$ or $\beta / \alpha(k<1$ always), where $\alpha$ and $\beta$ are electron and hole ionization coefficients. APD excess noise and response speed (gain bandwidth product) are determined by $\mathrm{k}$ value. For low noise and high speed applications, a small $\mathrm{k}$ value is critical. Common semiconductor materials excess noise factors are summarized in Figure 1.

There are several approaches to reduce the $\mathrm{k}$ value in InGaAs APDs [3]. Spectrolab has been developing InGaAs APDs with InAlAs material as a multiplication layer and InAlAs has a lower $\mathrm{k}$ value $(0.22)$ [2], than commonly used InP (0.5). In order to reduce the k-value further by engineering the impact ionization values of InAlAs based heterojunction multiplication layers and these structures were called $I^{2} E$ structures. In this approach, the multiplier layers of the APD consist of two different semiconductors with different ionization threshold energies. It turns out that the probability of avalanche events is higher in the semiconductor with lower ionization threshold energy. This preference translates into low noise because the avalanche events become less random. $k$ value of 0.1 has been demonstrated [4]. 
Excess noise values were measured on $I^{2} E$ APDs and the data are shown in Figure 2. The solid lines correspond to $k$ value of $0.1,0.15$, and 0.2 . The Spectrolab $I^{2} E$ APD shows $k$ value less than 0.1 at low gain regime due to the dead space effect. At high gain regime, the $\mathrm{k}$ value tends to fall on a value of 0.15 , which is considerably lower than the InP $(0.5)$ and the InAlAs (0.22). The measurement results are really encouraging for $I^{2} E$ approach. One advantage of $I^{2} E$ method is that it does not rely on the dead space effect, which could yield low $\mathrm{k}$ value with multiplier less than $0.1 \mu \mathrm{m}$ thick. The thin multiplier APDs tend to suffer from high dark current from tunneling. Also, it is difficult to grow uniform thin multiplier APD arrays. The Spectrolab I ${ }^{2} E$ APDs have a much thicker multiplier, which show very low dark current and good uniformity.

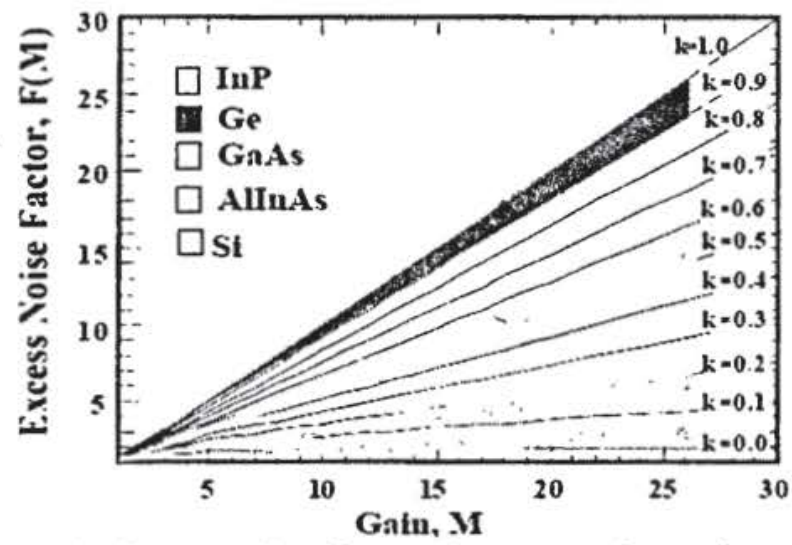

Figure 1. Excess noise factors for commonly used semiconductor materials.

One dimensional APD arrays were fabricated to characterize the wafer uniformity. The arrays had a pitch of $250 \mu \mathrm{m}$ and each individual APD had a mesa diameter of $75 \mu \mathrm{m}$. For 16 APDs, with a span of $4 \mathrm{~mm}$, the breakdown voltage variation was less than $200 \mathrm{mV}$ across the array. The variations on the photo current were the result of different incident light power on each APD during the measurement. The APDs showed low dark currents; at gain of 10, the average dark current of the APD array was $1.34 \mathrm{nA}$ with a standard deviation of $0.034 \mathrm{nA}$. Devices also showed high optical gain; an optical gain over 50 was achieved. We are pursuing new material structure design techniques to increase the gain to greater than 100 .

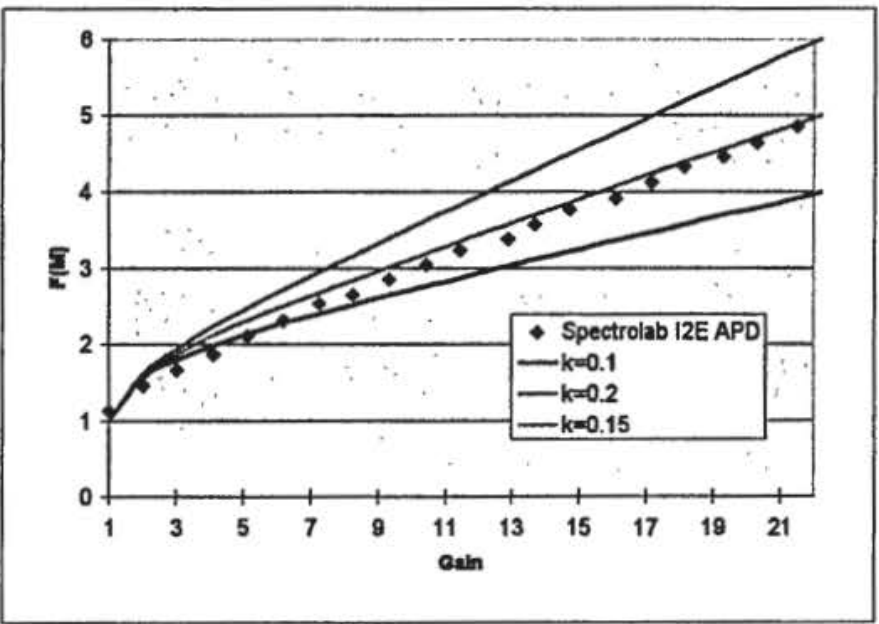

Figure 2. Excess noise data of $\mathrm{I}^{2} \mathrm{E}$ APD devices. 
At $1.06 \mu \mathrm{m}$, the APD quantum efficiency is $70 \%$ and the quantum efficiency can be further improved by depositing gold metal layer on the backside of APDs and we estimate quantum efficiency will increase to about $85 \%$ at $1.06 \mu \mathrm{m}$. Photoreceivers were built by integrating $\mathrm{I}^{2} \mathrm{E}$ APD devices with a low noise $\mathrm{GHz}$ transimpedance amplifier (TIA) and photoreceivers showed a bandwidth of $1 \mathrm{GHz}$ and an noise equivalent power (NEP) of less than $150 \mathrm{fW} / \mathrm{rt}(\mathrm{Hz})$ at room temperature. We believe the performance could be significantly improved by using a custom transimpedance amplifier rather than the present commercial low-cost TIA.

\section{INTENSIFIED PHOTODIODES}

The intensified photodiode (IPD) detector, (a.k.a hybrid photomultiplier tube (HPMT) or hybrid photodetector (HPD)), provides high-gain with low-noise suitable for single-photon sensitivity via electron bombardment of an avalanche diode. Advantages of the IPD include large detector area ( $1 \mathrm{~mm}$ for a single channel device), high maximum count rates $(>200 \mathrm{Mcps})$, high bandwidth $(\mathrm{GHz})$, low afterpulsing, and near room temperature operation. Further features include low dark noise, large dynamic range for full analog pulse received waveform preservation and photon number resolution. Operating principles and performance results of previous IPDs have been reported for use at visible [7], and near-infrared $[8,9,10]$, wavelengths. In recent work, we produced a 16-channel IPD with an InGaAsP photocathode for use at $1064 \mathrm{~nm}$ wavelength. Each channel has a $159 \mu \mathrm{m} \times 159 \mu \mathrm{m}$ sensitive detection area at the photocathode. Figure 3 shows the timing jitter (188 ps FWHM, 78 ps one-sigma) measurement results for our $0.8 \mu \mathrm{m}$ thick InGaAsP photocathode. We measured the timing jitter using two independent instruments 1) the Picoquant HydraHarp 400 multichannel scaler providing 188 ps FWHM measurement and an Agilent Model DSA91304A $(13 \mathrm{GHz} \mathrm{BW})$ oscilloscope that calculated the one sigma jitter directly from the waveform histogram as $78 \mathrm{ps}$. This is an upper limit on the timing jitter because it includes the photon timing uncertainty associated with the $100 \mathrm{ps}$ pulse width of the experimental test laser. For further context, a visible single-channel HPD with a $1 \mathrm{~mm}$ diameter photocathode has 28 ps timing jitter with $46 \%$ QE at $500 \mathrm{~nm}$ wavelength [3].

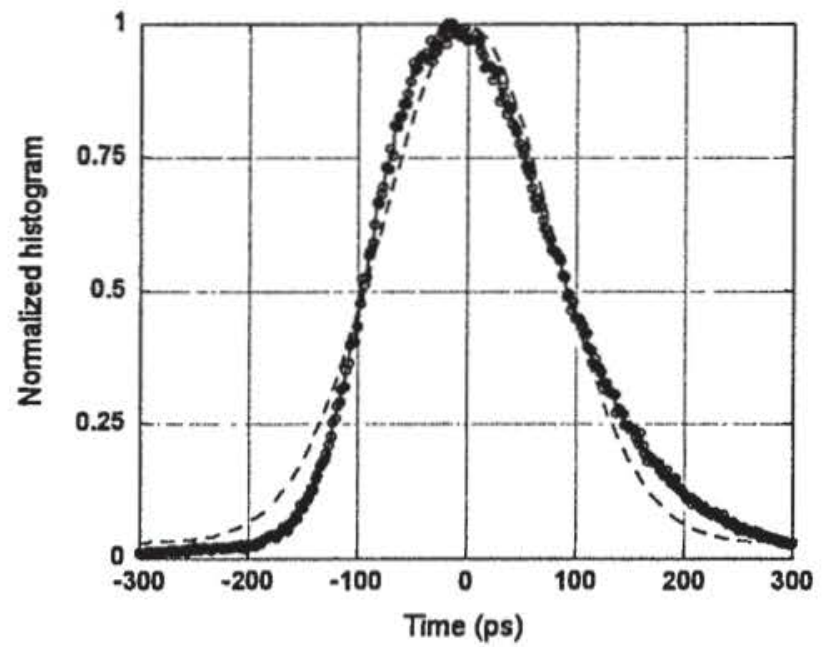

Fig. 3. Single-photon timing jitter measurement results for IPD with $0.8 \mu \mathrm{m}$ InGaAsP layer thickness. $\mathrm{FWHM}=188 \mathrm{ps}$. Dashed line is Gaussian fit with $\sigma=78 \mathrm{ps}$.

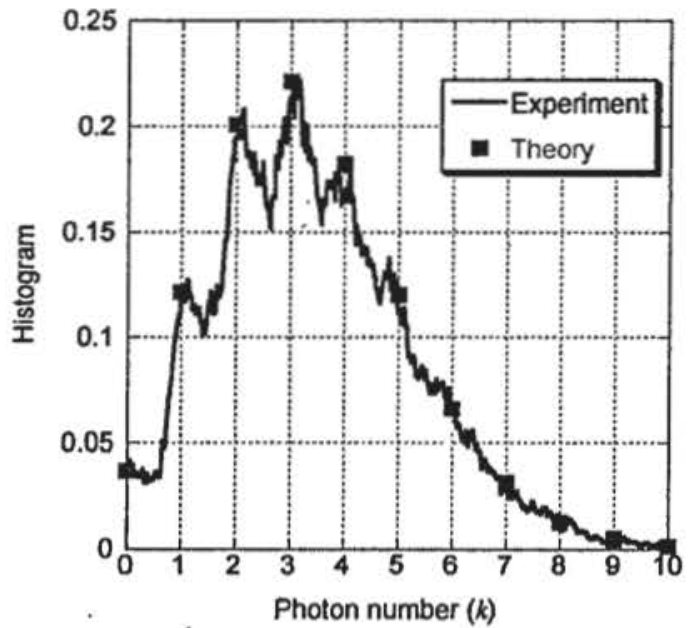

Fig. 4. Measured scaled histogram of the pulse height distribution and Poisson theory for $\lambda=3.3$

The single photon impulse response has a pulse width of $550 \mathrm{ps}$. The adjacent pixel cross talk was than $1.1 \%$ for any pair of adjacent pixels. The internal gain is $>10^{4}$ for each pixel. We measured reduction of dark counts per channel with decreasing temperature and predict less than $10 \mathrm{kcps}$ at $-20 \mathrm{C}$.

An excess noise factor of 1.2 was measured from the pulse height amplitude distribution. Figure 4 shows excellent agreement for two independent sets of measured photon number resolution (scaled pulse height distribution histogram) and the Poisson probability mass function $f(k, \lambda)=\frac{\lambda^{k} e^{-\lambda}}{k !}$ theory with $\lambda=3.3$. 


\section{DETECTORS FOR ICESat-2/ATLAS}

The Ice, Cloud and land Elevation Satellite 2/Advanced Topographic Laser Altimeter System (ICESat2/ATLAS) is planned for launch in December of 2014. ICESat2/ATLAS is a follow-on mission to the ICESat/GLAS mission whose primary purpose is to monitor the Earth polar ice sheets height and volume. ICESat/GLAS a linear-mode Si APD for the laser altimetry returns at $1064 \mathrm{~nm}$ and the photon-counting SPCMs to detect atmospheric backscatter returns at $532 \mathrm{~nm}$ from clouds and aerosols. In contrast, ICESat2/ATLAS will use photon-counting detectors and a high-repetition-rate $(10 \mathrm{kHz})$ laser to perform both the laser altimetry and atmospheric backscatter measurements at $532 \mathrm{~nm}$. The baseline ATLAS instrument design will use the $532 \mathrm{~nm}$ wavelength because of the insufficient maturity of space-qualifiable near-infrared single-photon-counting detectors. This is in spite of a preference for near-infrared wavelength operation. Near-infrared operation would provide a reduced solar background, double the number of photons for a given laser energy, lack of a complex doubling requirement and corresponding higher electrical efficiency and reduced thermal load. We have retained the InGaAsP near-infrared sensitive photocathode hybrid photomultiplier tube as an alternative. Key specifications for both are short dead time, maximum continuous count rate, detection efficiency, photon number resolution, timing jitter, radiation tolerance, power consumption, operating temperature and reliability. The ICESat2/ATLAS detector requirements are listed in Table 1.

Table 1. ICESat2/ATLAS detector requirements and Hamamatsu PMT performance

\begin{tabular}{|l|l|l|}
\hline Parameter & Requirement & $\begin{array}{l}\text { Measurements of } \\
\text { Hamamatsu 7600-M16 PMT }\end{array}$ \\
\hline $\begin{array}{l}\text { Technology Readiness Level } \\
\text { (TRL)/Space flight heritage }\end{array}$ & TRL 6 by November 2011 (orig. June 2011) & In process \\
\hline Photon Detection Efficiency & $\begin{array}{l}>15 \% \text { per pixel @ 532 nm } \\
>4 \% \text { per pixel @, 1064 nm }\end{array}$ & $\begin{array}{l}>15 \% \text { per pixel @ 532 nm } \\
>4 \% \text { per pixel @ 1064 nm }\end{array}$ \\
\hline Maximum count rate & $\begin{array}{l}12 \text { Mcps per full array (peak) } \\
2.5 \text { Mcps per full array (average) }\end{array}$ & $>160$ Mcps per full array \\
\hline Dead time & 2.5 ns maximum & $\begin{array}{l}2.5 \mathrm{~ns} \text { maximum } \\
\text { (from discriminator) }\end{array}$ \\
\hline Timing jitter & $380 \mathrm{ps} \mathrm{FWHM}$ & 340 ps FWHM \\
\hline Single-return intensity level & Up to 16 photon number resolution & $\begin{array}{l}16 \text { photon number resolution (with } \\
\text { lens array scheme) }\end{array}$ \\
\hline Dark Count rate & $<500$ Kcps per full array & $<32$ Kcps per full array \\
\hline Lifetime & $>3$ years, 5 year goal & $\begin{array}{l}\text { Lifetest in progress. } \\
\text { Heritage from PAMELA, AMS-02 } \\
\text { missions. }\end{array}$ \\
\hline
\end{tabular}

\subsection{Hamamatsu 7600-M16 photomultiplier tube}

Our baseline detector for use at $532 \mathrm{~nm}$ wavelength is the Hamamatsu Model 7600-M16 16-channel dynode-chain photomultiplier tube shown in Figure 5. This device was chosen because it has abundant reliability data and it has space flight heritage from the PAMELA' mission. In addition, this detector has undergone further qualification for the AMS02 mission $^{11}$ that will use 680 PMTs on the $\mathrm{RICH}^{12}$ instrument. The Model 7600 excess noise factor is too large to allow photon-number resolution. To achieve photon-number resolution and higher maximum count rate, each ground footprint single image return is spread over the photocathode array. The photon spatial and temporal distribution statistics allow the resolution of the photon-number as noted in References 8-11. This is the same technique we used on ICESat/GLAS except on ICESat-2/ATLAS we will use a lens array with a single multi-element anode detector rather than beamsplitters and multiple detectors. 


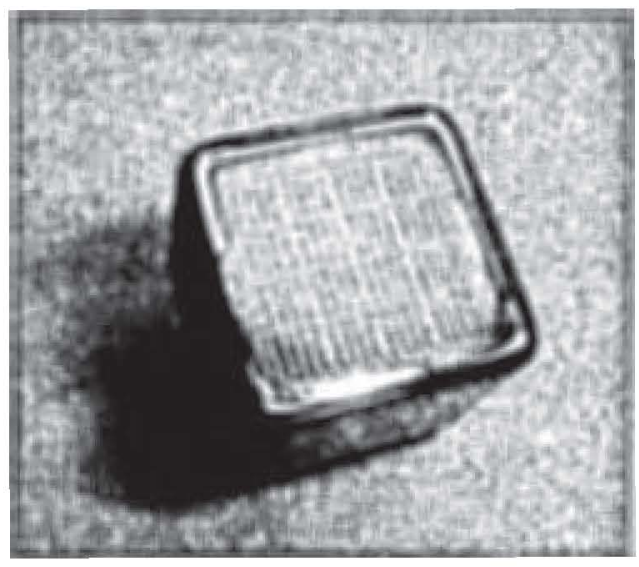

Fig. 5 Hamamatsu Model R7600-M16 16-channel dynode-chain photomultiplier tube.

\subsection{Intevac Intensified Photodiode}

We investigated using the Intevac hybrid photomultiplier tube with an InGaAsP photocathode as a possible alternative for ICESat2/ATLAS. To facilitate compatibility with our baseline detector approach, we studied the (4x4) 16-channel anode device. We investigated several design changes to improve the device reliability. Our results are still need further study.

\section{AIRBORNE LASER RECEIVER SYSTEM COMPARISON}

We plan to test both the 16 channel APD and IPD photoreceivers on the ALISTS instrument airborne flights. In preparation we have modeled the performance for the ALIST Swath Mapper instrument using a $10 \mathrm{kHz}$ repetition rate $1030 \mathrm{~nm}$ Yb:YAG laser with $1 \mathrm{~ns}$ pulsewidth at $10 \mathrm{~km}$ altitude with a $5 \mathrm{~m}$ ground spot diameter, 3 degree surface slope, 7" diameter receiver telescope and 80 degree Sun angle.

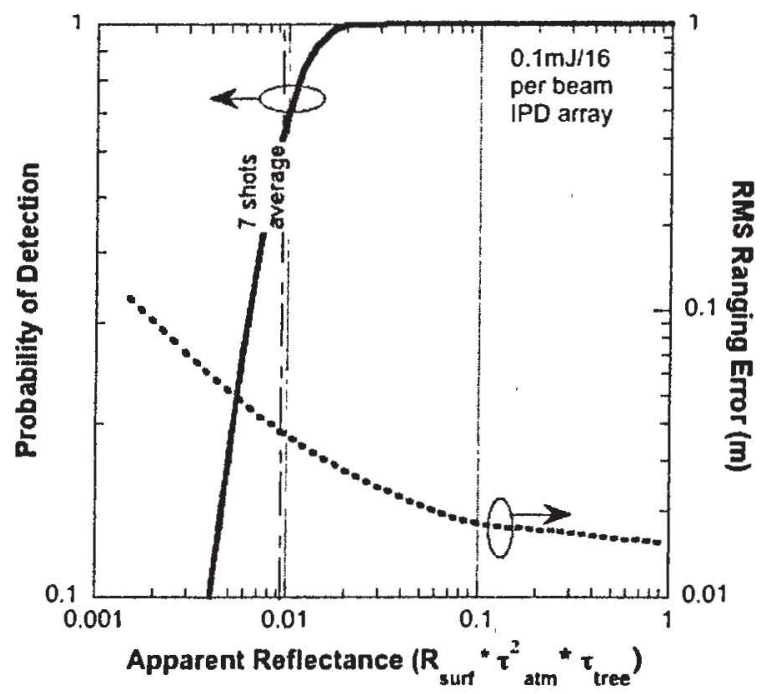

Fig. 6. ALIST Swath Mapper Performance with IPD receiver.

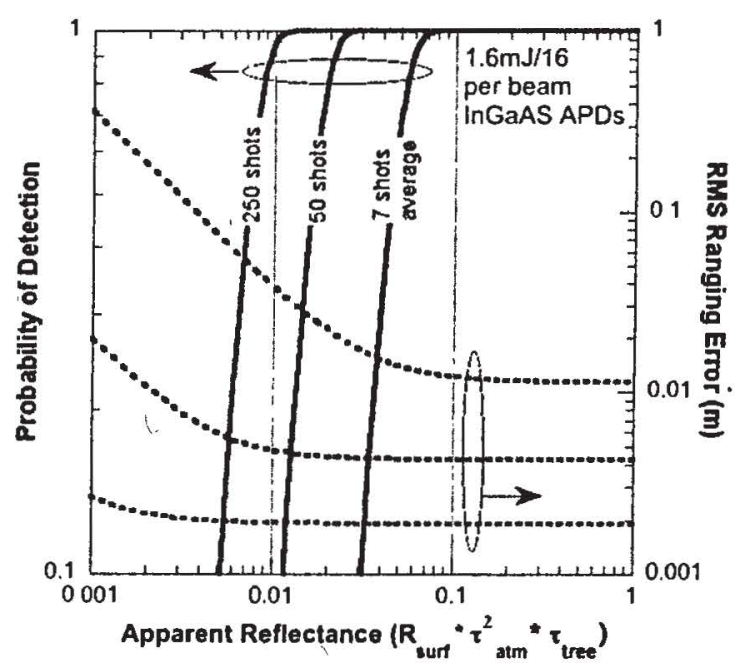

Fig. 7. ALIST Swath Mapper Performance with I2E APD receiver. 
Figures 6 and 7 show the numerical model result of the probability of detection (left ordinate - solid curves) and the RMS ranging error (right ordinate - dashed curves) vs. the apparent surface reflectivity for the IPD (with a $0.1 \mathrm{~mJ}$ laser energy and 7 shot average)) and $\mathrm{I}^{2} \mathrm{E}$ APD (with $1.6 \mathrm{~mJ}$ laser energy and 250 shot average)) respectively. The system operating points of interest for comparison are circled with arrows indicating the appropriate axis. The IPD shows far superior laser receiver performance. However, the $I^{2} E$ APD receiver at present has far superior component reliability. Both laser receivers can achieve the same desired performance with the associated laser energies and averaging.

\section{ACKNOWLEDGMENT}

We greatly appreciate the support of the NASA ESTO ACT, ESTO IIP and ICESat-2/ATLAS project that is funding this work.

\section{REFERENCES}

[1] Earth Science and Applications from Space: National Imperatives for the Next Decade and Beyond, National Research Council of the National academies, The National Academies Pres, Washington D.C., (2007).

[2] M.A. Krainak, X. Sun, G. Yang, L.R. Miko, and J.B. Abshire, "Photon detectors with large dynamic range and at near-infrared wavelength for direct detection space lidars" Proc. SPIE 7320, 732005 (2009).

[3] M.A. Krainak, X. Sun, G. Yang, and W. Lu, "Comparison of linear-mode avalanche photodiode lidar receivers for use at one-micron wavelength" Proc. SPIE 7681, 76810Y (2010).

[4] F. Laforce "Low noise optical receiver using Si APD" Proc. SPIE 7212, 721210 (2009).

[5] C.S. Gardner, "Target signatures for laser altimeters: an analysis" Applied Optics Vol. 21, No. 3 pp. 448-453 February 1982.

[6] A. W. Yu, D. J. Harding, M. Krainak, J. B. Abshire, X. Sun, J. F Cavanaugh, S. R. Valett, L. Ramos-Izquierdo, "Efficient Swath mapping Laser Altimeter Instrument Incubator Program American Geophysical Union, Abstract \#EP44B-01 (2010).

[7] A. Fukasawa, J. Haba, A. Kageyama, H. Nakazawa, and M. Suyama "High Speed HPD for Photon Counting," IEEE Transactions On Nuclear Science 55, 758-762 (2008).

[8] X. Sun, M. A. Krainak, W. E. Hasselbrack, D. F. Sykora, R. La Rue, "Single photon counting at 950 to $1300 \mathrm{~nm}$ using InGaAsP photocathode - GaAs avalanche photodiode hybrid photomultiplier tubes," Journal of Modern Optics, 56, 284-295, (2009).

[9] R. A. La Rue, K. A. Costello, G. A. Davis, J. P. Edgecumbe, V. W. Aebi, "Photon Counting III-V Hybrid Photomultipliers Using Transmission Mode Photocathodes," IEEE Trans. Electron Devices 44, 672-678 (1997).

[10] R. A. La Rue, G. A. Davis, D. Pudvay, K. A. Costello, V. W. Aebi, "Photon Counting 1060-nm Hybrid Photomultiplier with High Quantum Efficiency," IEEE Electron Device Lett. 20, 126-128 (1999).

[11] Alpat, B., "Alpha Magnetic Spectrometer (AMS02) experiment on the International Space Station (ISS)", Nuclear Science and Techniques, Vol.14 No.3 pp. 182-194 (2003)

[12] Vargas-Treviho, M., et al., "A Test Bench for the AMS-RICH Photodetector Modules" IMTC 2004 Instrumentation and Measurement Technology Conference Coma, Italy. (2004) 